\title{
Dissecting and engineering of the TetR family regulator SACE_7301 for enhanced erythromycin production in Saccharopolyspora erythraea
}

Hang Wu' ${ }^{1}$, Meng Chen ${ }^{1}$, Yongrong Mao ${ }^{1}$, Weiwei Li ${ }^{1}$, Jingtao Liu ${ }^{1,4}$, Xunduan Huang ${ }^{1}$, Ying Zhou ${ }^{3}$, Bang-Ce Ye ${ }^{3}$, Lixin Zhang ${ }^{1,2^{*}}$, David T Weaver ${ }^{1 *}$ and Buchang Zhang ${ }^{1 *}$

\begin{abstract}
Background: Saccharopolyspora erythraea was extensively utilized for the industrial-scale production of erythromycin A (Er-A), a macrolide antibiotic commonly used in human medicine. Yet, S. erythraea lacks regulatory genes in the erythromycin biosynthetic gene (ery) cluster, hampering efforts to enhance Er-A production via the engineering of regulatory genes.

Results: By the chromosome gene inactivation technique based on homologous recombination with linearized DNA fragments, we have inactivated a number of candidate TetR family transcriptional regulators (TFRs) and identified one TFR (SACE_7301) positively controlling erythromycin biosynthesis in S. erythraea A226. qRT-PCR and EMSA analyses demonstrated that SACE_7301 activated the transcription of erythromycin biosynthetic gene eryAl and the resistance gene ermE by interacting with their promoter regions with low affinities, similar to BldD (SACE_2077) previously identified to regulate erythromycin biosynthesis and morphological differentiation. Therefore, we designed a strategy for overexpressing SACE_7301 with 1 to 3 extra copies under the control of PermE* in A226. Following up-regulated transcriptional expression of SACE_7301, eryAl and ermE, the SACE_7301-overexpressed strains all increased Er-A production over A226 proportional to the number of copies. Likewise, when SACE_7301 was overexpressed in an industrial S. erythraea WB strain, Er-A yields of the mutants WB/7301, WB/2×7301 and WB/3×7301 were respectively increased by $17 \%, 29 \%$ and $42 \%$ relative to that of WB. In a $5 \mathrm{~L}$ fermentor, Er-A accumulation increased to 4,230 mg/L with the highest-yield strain WB/3×7301, an approximately 27\% production improvement over WB (3,322 mg/L).

Conclusions: We have identified and characterized a TFR, SACE_7301, in S. erythraea that positively regulated erythromycin biosynthesis, and overexpression of SACE_7301 in wild-type and industrial S. erythraea strains enhanced Er-A yields. This study markedly improves our understanding of the unusual regulatory mechanism of erythromycin biosynthesis, and provides a novel strategy towards Er-A overproduction by engineering transcriptional regulators of S. erythraea.
\end{abstract}

Keywords: Saccharopolyspora erythraea, Erythromycin, SACE_7301, TetR family regulator, Gene overexpression, Metabolic engineering

\footnotetext{
* Correspondence: zhanglixin@im.ac.cn; David.weaver.@gmail.com; zhbc@ahu.edu.cn ${ }^{1}$ Institute of Health Sciences, School of Life Sciences, Anhui University, Hefei 230601, China

${ }^{2}$ CAS Key Laboratory of Pathogenic Microbiology \& Immunology, Institute of Microbiology, Chinese Academy of Sciences, Beijing 100101, China Full list of author information is available at the end of the article
}

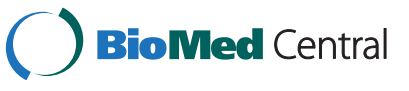

(c) 2014 Wu et al.; licensee BioMed Central Ltd. This is an Open Access article distributed under the terms of the Creative Commons Attribution License (http://creativecommons.org/licenses/by/4.0), which permits unrestricted use, distribution, and reproduction in any medium, provided the original work is properly credited. The Creative Commons Public Domain Dedication waiver (http://creativecommons.org/publicdomain/zero/1.0/) applies to the data made available in this article unless otherwise stated. 


\section{Background}

Saccharopolyspora erythraea, a Gram-positive industrial actinomycetes, produces a valuable macrolide antibiotic erythromycin A (Er-A). Er-A and its semi-synthetic derivatives, such as clarithromycin, azithromycin, dirithromycin, roxithromycin, and telithromycin, are widely used in medicine to treat infections caused by pathogenic Grampositive bacteria [1]. Considering its high industrial and medical importance, enhancement of Er-A production has been performed by metabolic engineering or classical mutagenesis methods over the past 60 years [2-4]. Also, as a model actinomycete system, S. erythraea has been used for studying the biosynthesis and combinatorial biosynthesis of polyketide antibiotics, which are synthesized by modular type I polyketide synthase (PKS) [5,6]. The erythromycin biosynthetic pathway has been investigated by genetic and biochemical approaches $[7,8]$. The erythromycin biosynthetic gene (ery) cluster contains 20 genes arranged in four major polycistronic units, spanning over $60 \mathrm{~kb}$ of DNA [9]. Unusually, the ery cluster lacks any regulatory genes in S. erythraea, hampering efforts to improve Er-A production by modulating gene regulation pathways.

Based on a DNA microarray strategy, the industrial overproducer strain was found to express the entire ery cluster several days longer than the wild-type strain, suggesting that there exist some regulators for controlling erythromycin biosynthesis [10]. In vitro and in vivo approaches led to the identification of BldD (SACE_2077), a key developmental regulator in actinomycetes [11], controlling erythromycin biosynthesis and morphological differentiation in S. erythraea [12]. BldD was found to bind to the promoter regions of ery cluster. However, the action of BldD on the promoters of ery genes was much weaker than on its own promoter [12]. Recently, a putative regulatory protein, SACE_5599, was proved to positively control erythromycin production and morphological differentiation in S. erythraea [13]. Nevertheless, the knowledge of molecular regulatory mechanisms controlling erythromycin biosynthesis remains limited.

The TetR family transcriptional regulators (TFRs), containing an N-terminal HTH DNA-binding motif and a C-terminal ligand recognition domain, are widely distributed in bacteria, playing important roles in antibiotic biosynthesis, efflux pumps, osmotic stress, and other functions [14]. TFRs regulate the biosynthesis of multiple antibiotics or morphogenesis of actinomycetes [15-28], suggesting that certain TRFs from S. erythraea are candidates for a role in erythromycin biosynthesis. With the availability of the complete genome sequence, 101 putative TFRs in S. erythraea were inferred [29]. Based on the homologous recombination with linearized DNA fragments to inactivate specific genes, a number of the S. erythraea TFRs were examined, resulting in the identification of two TFRs (SACE_7040 and SACE_0012) having an association with morphological differentiation [30,31], and one TFR (SACE_3986) negatively controlling the erythromycin biosynthesis [32]. Nevertheless, functions of most TFRs in S. erythraea remain to be elucidated.

In this study, we identified and characterized a novel TFR (SACE_7301) that positively regulated erythromycin biosynthesis. SACE_7301 was found to increase the transcription of erythromycin biosynthetic gene eryAI and the resistance gene ermE by binding to their promoter regions in S. erythraea. Further overexpression of SACE_7301 led to enhanced Er-A titers in the wild-type S. erythraea A226 and the high-yield industrial strain WB.

\section{Results}

\section{SACE_7301 positively regulates the erythromycin} biosynthesis

Since extensive investigations have proved that TFRs are involved in the antibiotic biosynthesis in actinomycetes through gene inactivation and bioassay experiments [33], we have identified several TFRs pertinent to erythromycin production in $S$. erythraea, including the repressor SACE_3986 [32], and the activator SACE_7301 currently studied. By homologous recombination of linearized fragments (Figure 1A), SACE_7301 was replaced by the thiostrepton resistance gene (tsr) in S. erythraea A226, and the desired mutant, named as $\triangle S A C E_{-} 7301$, was isolated and confirmed by PCR analysis (Figure 1B).

Erythromycin titers of A226 and $\triangle S A C E_{-} 7301$ were estimated by fermentation and bioassay. $\triangle S A C E \_7301$ displayed lower inhibitory activity against Bacillus subtilis relative to A226, but had slightly higher levels than the bldD deletion mutant (Figure 1C), a previously identified regulator of erythromycin biosynthesis [12]. Furthermore, A226 and $\triangle S A C E \_7301$ were cultivated at $30^{\circ} \mathrm{C}$ for up to 6 days in the $\mathrm{R} 5$ liquid fermentation medium, and the extracts of those cultures were analyzed by HPLC. Compared with the parental strain A226, $\triangle S A C E \_7301$ had a $38 \%$ reduction in the Er-A yield in 6 day fermentation period (from $58 \mathrm{mg} / \mathrm{L}$ to $36 \mathrm{mg} / \mathrm{L}$ ). (Figure 1D). However, the two strains had comparable growth rates and cell densities (Figure 1E). Thus, SACE_7301 is postulated to positively regulate erythromycin production in S. erythraea.

In order to exclude the possibility that the decreased erythromycin production was due to a random mutation in other chromosome loci of S. erythraea, $\triangle S A C E \_7301$ was complemented with a copy of $S A C E$ _7301 (pZMW7301). The Er-A yield of the complemented strain $\triangle S A C E \_7301 /$ pZMW7301 was nearly recovered to the parental level (Figure 1F). To further substantiate the role of SACE_7301 as an activator for erythromycin production, we introduced pZMW and pZMW7301 into A226, obtaining the 


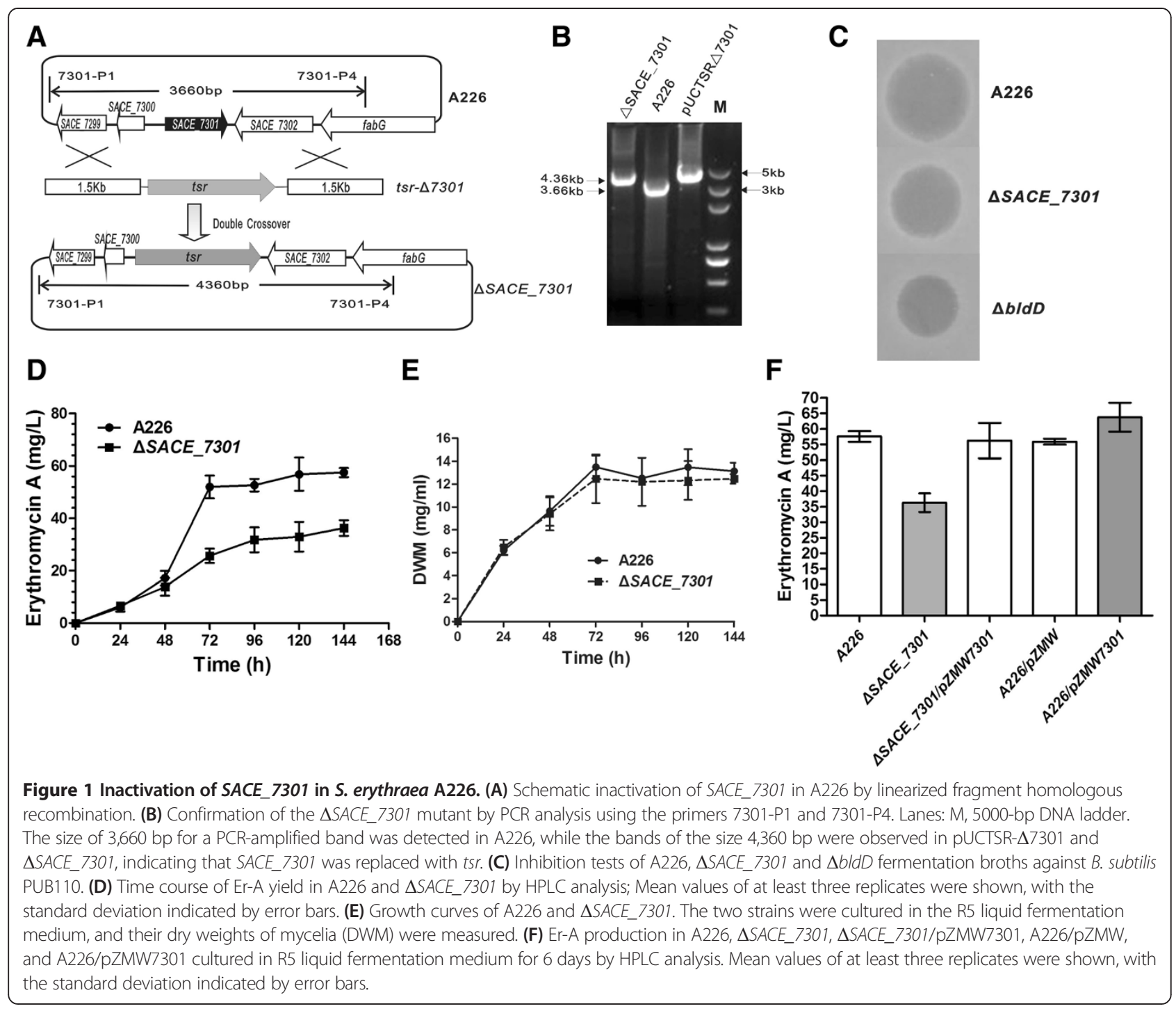

control strain A226/pZMW and the SACE_7301-overexpressed strain A226/pZMW7301, respectively. As detected by HPLC analysis, the Er-A yield of A226/pZMW-7301 was $64 \mathrm{mg} / \mathrm{L}$, that was $10 \%$ and $14 \%$ increased relative to those of A226 (58 mg/L) and A226/pZMW (56 mg/L) respectively (Figure 1F). Taken together, our results verified that SACE_7301 acted as a positive regulator controlling the erythromycin production in S. erythraea.

\section{SACE_7301 is not involved in the morphological differentiation of S. erythraea}

Previously, BldD and SACE_5599 were identified to concurrently regulate erythromycin biosynthesis and morphological differentiation in S. erythraea [12,13]. In order to discern whether SACE_7301 was also associated with the morphological differentiation, we inoculated A226 and its derivative mutants on R3M agar medium for observing sporulation. $\triangle S A C E \_7301, \triangle S A C E \_7301 /$ pZMW7301 and
A226/pZMW7301 on R3M plates were identical to A226 in the rate of aerial mycelia formation, whereas $\Delta b l d D$ showed the defect of aerial hyphae as expected (data not shown). These results further substantiate that SACE_7301 acts exclusively as a positive regulator for the synthesis of erythromycin in S. erythraea.

\section{Overexpression of SACE_7301 increases the transcription of eryAl and ermE}

To investigate the effect of $S A C E \_7301$ overexpression on the transcription of ery genes, we chose erythromycin biosynthetic gene eryAI (encoding polyketide synthase I) and the resistance gene ermE (encoding rRNA methyltransferase) for transcriptional comparison between control strain A226/pZMW and overexpressed strain A226/ pZMW7301 on the second or fourth day of growth. When grown for 4 days, A226/pZMW7301 exhibited 2.2- and 1.7- folds transcription increases detected by 
qRT-PCR for eryAI and ermE in comparison to A226/ pZMW (Figure 2B). Similar transcriptional levels of eryAI and ermE were found for the two strains in 2-day growth (Figure 2A). The data supports the interpretation that SACE_7301 plays a positive role in the erythromycin biosynthesis by increasing the transcription of several structural and resistance genes.

\section{SACE_7301 binds to the promoter regions of eryAl and ermE}

TetR family members often regulate the transcription of target genes by specifically binding to their promoters [33]. To examine whether SACE_7301 might directly regulate the transcription of eryAI and ermE, a full-length SACE_7301 gene was expressed in Escherichia coli BL21 (DE3) using pET22b, and the purified SACE_7301 protein fused with His tag (Additional file 1: Figure S1) was used for in vitro binding experiments by EMSA.

6-FAM labeled eryAI and ermE promoter DNA fragments were respectively mixed with purified $\mathrm{His}_{6}$-tagged SACE_7301. At concentrations equal or greater than $1 \mu \mathrm{M}$ of SACE_7301, mobility shifts were evident. Meantime, it was found that a 50-fold excess of unlabeled probes markedly competed with labeled probes for binding to SACE_7301 (Figure 3A-B). As a negative control, a non-specific DNA, poly dIdC, was used to compete with the 6-FAM labeled probes, and the shifted bands did not disappear (Figure 3A-B), confirming that SACE _7301 specifically bound to the promoter regions of eryAI and ermE.

To evaluate the relative affinity of SACE_7301 to abovementioned probes, we expressed recombinant BldD protein in E. coli BL21 (DE3), and purified it with $\mathrm{His}_{6}$-tag (Additional file 1: Figure S1). EMSAs showed that the concentrations of BldD shifting the two promoter probes of eryAI and ermE were similar to that of SACE_7301 (Figure 3C-D), indicating that SACE_7301 and BldD had the same degree of affinities to the two promoters. Therefore, the combined data indicates that SACE_7301 up-regulates the transcription of eryAI and ermE by directly interacting with their promoter regions.

\section{Effects of SACE_7301 overexpression on erythromycin biosynthesis in S. erythraea A226}

As overexpression of $S A C E \_7301$ with an extra copy displayed an increase of Er-A production in S. erythraea A226 as described above, we proposed that increased copy numbers of $S A C E \_7301$ would lead to additional increases in Er-A levels. SACE_7301 under the control of PermE* was cloned into pSET152, creating 1, 2, or 3 copies of $S A C E \_7301$, introduced into A226 (Figure 4A), to generate A226/7301, A226/2×7301 and A226/3×7301 strains respectively. By fermentation and HPLC analysis, the three strains all presented Er-A product improvement (Figure 4B). Furthermore, the Er-A production exhibited a positive correlation with the copy number of $S A C E$ _7301, reaching the highest 1.44 folds with 3 copies (Figure 4B). When cultured in the R5 liquid medium, these strains had comparable growth rates and cell densities (Additional file 1: Figure S2), suggesting that the enhanced erythromycin yield in SACE_7301-overexpressed strains was not caused by the increase of biomass.

In addition, qRT-PCR was used to compare the transcriptional levels of $S A C E \_7301$, eryAI and ermE between A226 and its $S A C E_{-} 7301$-overexpressed strains. With the increase of $S A C E \_7301$ copy number, the transcripts of $S A C E$ _7301 in A226/7301, A226/2×7301 and A226/3×7301 were found to be up-regulated by 4.6-, 8.4- and 16.5-folds relative to that of A226 (Figure 4C). Likewise, the transcripts of eryAI and ermE in those mutants were gradually increased compared to the original level (Figure 4C). These results indicated that overexpression of $S A C E \_7301$ with 1 to 3 extra copies in S. erythraea associated with a stepwise increase of Er-A production by promoting the transcription of eryAI and ermE. In conjunction with RNA changes, A226/3×7301 also demonstrated increased resistance to erythromycin
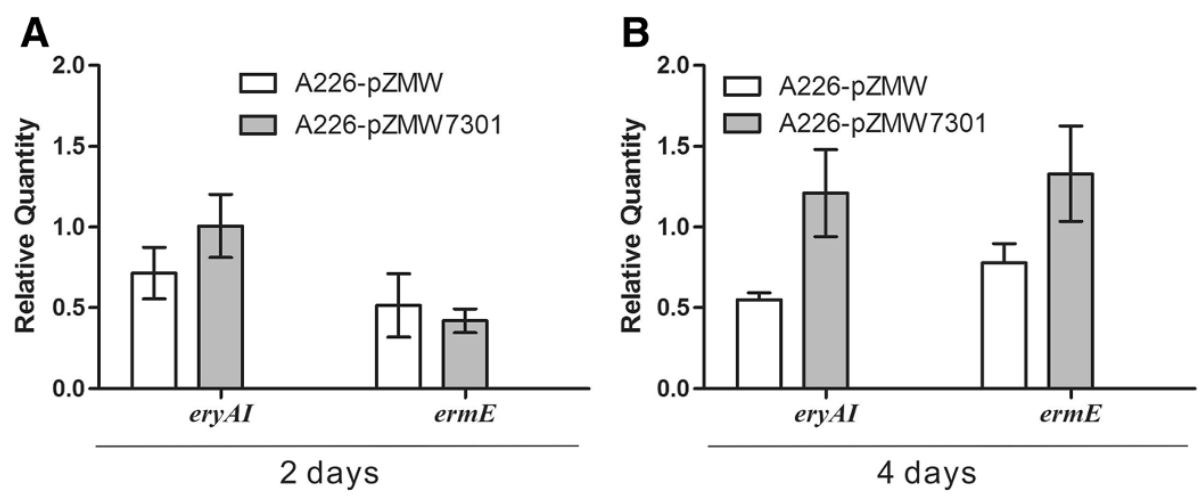

Figure 2 Effects of $S A C E \_7301$ overexpression on transcriptional levels of eryAl and ermE. qRT-PCR was used to quantify the amounts of transcripts produced by A226/pZMW, and A226/pZMW7301 cultured in R5 fermentation medium for 2 (A) or 4 days (B). Mean values of at least three replicates were shown, with the standard deviation indicated by error bars. 


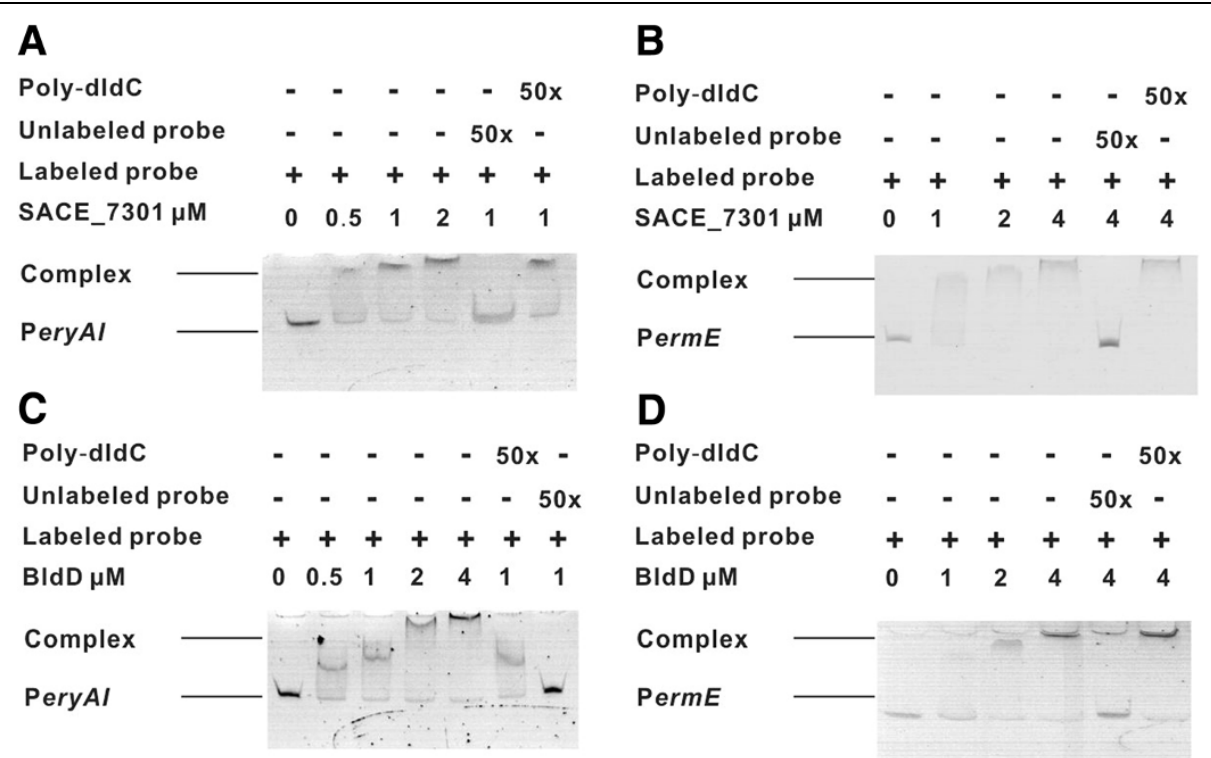

Figure 3 Electrophoretic mobility shift assays (EMSAs) with the promoter regions of eryAl or ermE and purified His ${ }_{6}$-tagged SACE_7301 or His $_{6}$-tagged BIdD. (A) EMSAs with recombinant SACE_7301 protein and PeryAl. (B) EMSAs with recombinant SACE_7301 protein and PermE. (C) EMSAs with recombinant BIdD protein and PeryAl. (D) EMSAs with recombinant BldD protein and PermE. Each of the lanes contained $10 \mathrm{ng}$ DNA. Results were one representative example of at least three replicates.

over A226 when cultured for 2 days with addition of greater than $160 \mu \mathrm{g} / \mathrm{ml}$ erythromycin (Additional file 1: Figure S3).

Also, we cultured A226/3×7301 in the R5 liquid medium for 6 days without addition of apramycin, and found that the size of the gene expression cassette containing the 3 copies of SACE 7301 from plasmid pSET152-3×7301 did not change by PCR analysis (Additional file 1: Figure S4), indicating that the engineered strain had a stably inserted SACE_7301.

\section{Improved Er-A production by overexpression of SACE_7301 in an industrial strain WB}

To examine the applicability of overexpression of $S A C E \_7301$ for enhancing erythromycin production in industrially-relevant strains, $S A C E \_7301$ was introduced into an industrial S. erythraea WB strain with 1,2 or 3 extra copies under the control of PermE*, and the desired mutants $\mathrm{WB} / 7301, \mathrm{WB} / 2 \times 7301$ and $\mathrm{WB} / 3 \times 7301$ were obtained. In flask experiments with an industrial medium for 6 days, the Er-A yields of WB/7301 (685 $\mathrm{mg} / \mathrm{L}), \mathrm{WB} / 2 \times 7301(755 \mathrm{mg} / \mathrm{L})$ and $\mathrm{WB} / 3 \times 7301(832$ $\mathrm{mg} / \mathrm{L}$ ) were respectively increased by $17 \%, 29 \%$, and $42 \%$ in comparison to that of WB $(586 \mathrm{mg} / \mathrm{L})$ (Figure $5 \mathrm{~A})$. Likewise, no significant change of cell growth was detected for these strains in comparison (Additional file 1: Figure S2).

We also tested the reproducibility of one of the engineered strains in a larger scale by selecting the highestyield strain $\mathrm{WB} / 3 \times 7301$. Following 8 -day fermentation at a $5 \mathrm{~L}$ fermentor scale, $\mathrm{WB} / 3 \times 7301$ exhibited a strikingly higher Er-A yield (4,230 $\mathrm{mg} / \mathrm{L})$, that was $27 \%$ greater than WB $(3,322 \mathrm{mg} / \mathrm{L})$. Since this strain maintains the characteristics of its parental strain, we conclude that the additional Er-A production from SACE_7301-engineered industrial strains will be of significant commercial value.

\section{Discussion}

TFRs form homodimers that generally act as transcriptional repressors to regulate the transcription of genes involved in antibiotic biosynthesis or resistance [34], but little is known about the TFR as a positive regulator for controlling the antibiotic biosynthesis in actinomycetes. Recently, the TetR family regulatory gene gouR, situated in the gene cluster of gougerotin biosynthesis, was characterized to be a positive regulator for modulating the gougerotin production by coordinating its biosynthesis and export in Streptomyces graminearus [27]. In this work, we identified a novel TFR (SACE_7301) from $S$. erythraea, and proved that it played a positive role in regulating erythromycin biosynthesis.

SACE_7301 was shown to specifically bind to the promoter regions of eryAI and ermE in vitro, and enhance their transcription. SACE_7301, located approximately $8.1 \mathrm{Mb}$ in the chromosome of S. erythraea, is not closely positioned with the ery cluster (GenBank Accession No. NC-009142. 778,214-832,825 nt, $0.8 \mathrm{Mb}$ ) and therefore it became evident that the gene product of SACE_7301 might function as a trans-acting factor remotely regulating erythromycin biosynthesis. This regulation pattern 

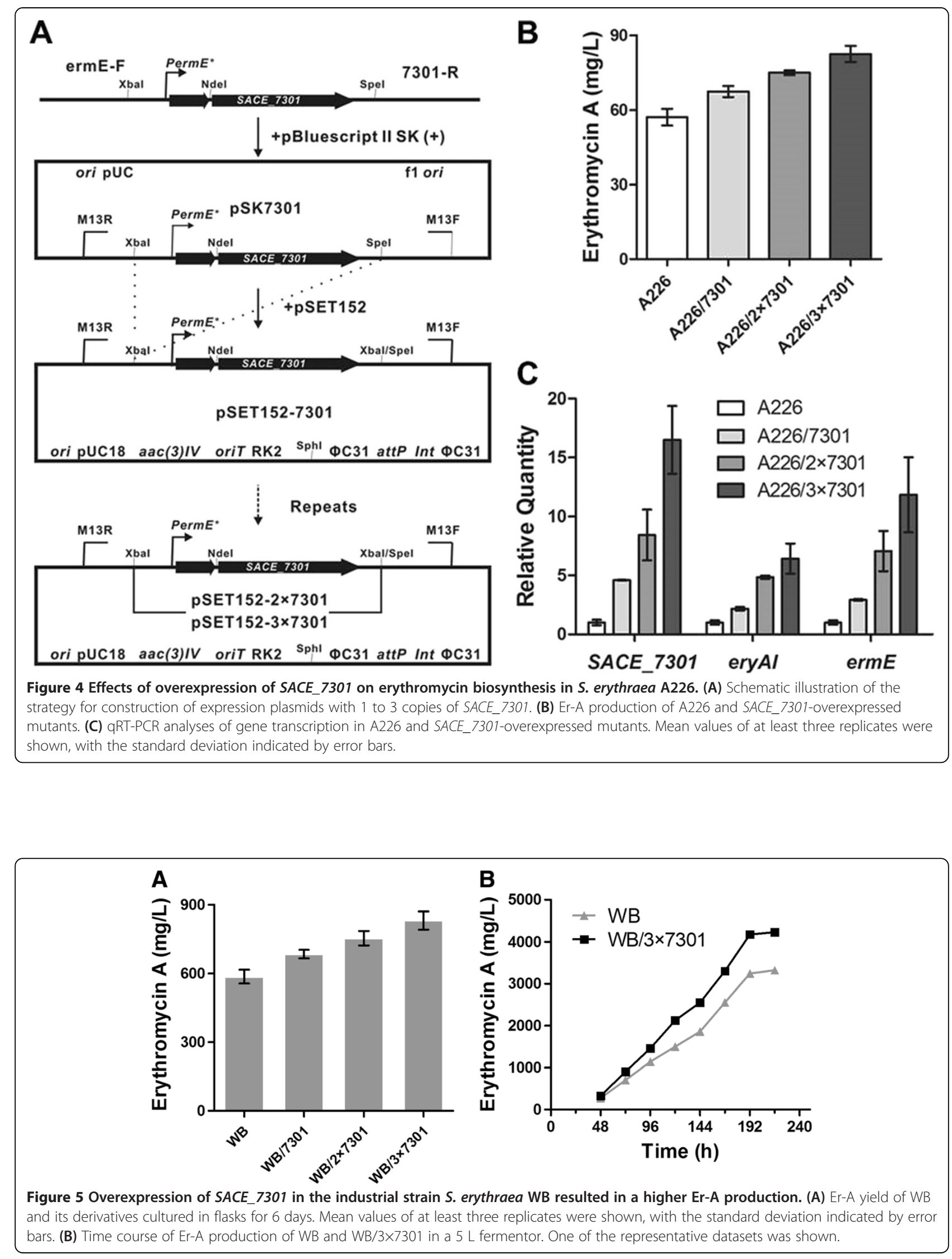
may be similar to that of BldD, a previously identified regulator for erythromycin biosynthesis [12]. As SACE_7301 exhibited low affinities to eryA and ermE promoters, it was difficult to determine the tight binding sites through DNase I footprinting assay. On the other hand, moderate increase in Er-A production was achieved by overexpression of SACE_7301 with an extra copy, similar to BldD in S. erythraea A226 [35]. These data indicate that SACE_7301 is likely a positive regulator that directly acts on the promoters of ery genes.

Through phylogenetic analysis [34], SACE_7301 homologs are distributed in rare actinomycetes, such as KUTG_05296 from Kutzneria sp. 744 (62\% identity), Amir_6979 from Actinosynnema mirum ATCC29888 (63\% identity), BN6_83740 from Saccharothrix espanaensis ATCC51144 (60\% identity) (Additional file 1: Figure S5). However, since none of these TFRs has been uncovered in actinomycetes, it potentially signifies a novel regulatory mechanism in actinomycetes, such as how it functions with its ligand [36].

To further improve the production of erythromycin, an overexpression tactic was designed and utilized for overexpressing SACE_7301 under PermE* in wild-type strain A226 and industrial overproducer WB. Along with the increase of $S A C E \_7301$ copy number, $S A C E \_7301$, ery $A I$ and $e r m E$ were significantly up-regulated, and ErA yields of those mutants rose step by step. However, these changes in transcription level do not linearly correspond to the changes in Er-A production. A similar result was also reported for the TetR family regulator GouR in S. graminearus [27].

Overexpression of bldD or SACE_5599 could improve erythromycin production in the wild-type $S$. erythraea strain, but not in the industrial overproducer [13]. Considering that WB is already an industrial strain, it is likely that overexpression of SACE_7301 will have commercial value when applied in other erythromycin high-yield $S$. erythraea strains. In other examples, the overexpression approach was utilized to engineer the halogenase gene $c t c P$, resulting in improvement of chlortetracycline production in industrial S. aureofaciens [37]. Overexpression of tailor genes eryK and ery $G$ was also performed to enhance the production and purity of Er-A in an industrial S. erythraea [4]. To our knowledge, this is the first report for tandemly expressing transcriptional regulators to enhance antibiotic production in actinomycetes. We anticipate that the strategy of overexpression of additional regulators will be generally applicable for improvement of other antibiotics in industry.

\section{Conclusion}

The novel TFR SACE_7301 positively regulates erythromycin biosynthesis in S. erythraea, and it activated the transcription of erythromycin biosynthetic gene eryAI and the resistance gene ermE by interacting with their promoters.
Furthermore, when $S A C E$ _ 7301 was overexpressed in wildtype or industrial S. erythraea strains with 1 to 3 extra copies, Er-A yields had stepwise enhancement in comparison to the parental strains. Our data here form a new understanding of the unusual regulatory mechanism of erythromycin biosynthesis, and provide a valuable means to improve erythromycin production.

\section{Materials and methods}

Strains, plasmids, and culture conditions

All strains and plasmids used in this study are listed in Table 1. S. erythraea and its derivatives were grown either in TSB liquid medium for seed culture, DNA extraction and protoplast preparation, or on R3M agar medium for protoplast regeneration or phenotypic observation with thiostrepton or apramycin when appropriate [38,39]. E. coli and B. subtilis strains were cultured in Luria-Bertani (LB) liquid medium or on LB plates at $37^{\circ} \mathrm{C}$ [40]. The general techniques in $E$. coli and S. erythraea were performed as described $[38,40]$.

\section{Gene inactivation, complementation and overexpression of SACE_7301}

With S. erythraea A226 genomic DNA as a template, two 1.5-kb DNA fragments flanking the $S A C E \_7301$ gene were amplified by PCR using the primer pairs 7301-P1/7301-P2 and 7301-P3/7301-P4 (Table 2). The two PCR products were respectively digested with EcoRI/KpnI and XbaI/ HindIII, and ligated into the corresponding sites of pUCTSR [30], obtaining pUCTSR- $\Delta 7301$. By the homologous recombination with linearized fragments [41], the SACE_7301 gene of S. erythraea A226 was replaced with the thiostrepton resistance gene $(t s r)$. The desired thiostrepton-resistant mutant, named $\triangle S A C E_{-} 7301$, was further confirmed by PCR analysis using the primers 7301-P1/7301-P4 (Table 2).

Using the primers 7301-P5 and 7301-P6 (Table 2), a 0.66kb DNA fragment containing a full-length $S A C E \_7301$ was amplified by PCR with the genomic DNA of A226 as a template. The PCR product was cleaved with NdeI/EcoRV, and inserted into the corresponding sites of integrative plasmid pZMW [42], yielding pZMW-7301. By PEG-mediated protoplast transformation, pZMW7301 was introduced into the $\triangle S A C E$ _7301 mutant and the parental strain A226, respectively. The complemented strain $\triangle S A C E_{-} 7301 /$ pZMW7301 and overexpression strain A226/pZMW7301 were obtained by apramycin resistance screening and confirmed by PCR analysis with the primers apr-F and apr-R (Table 2).

\section{Overexpression of SACE_7301 in S. erythraea}

In order to introduce 1 to 3 extra copies of $S A C E \_7301$ into the chromosome of $S$. erythraea, the combined DNA fragment containing PermE* and SACE_7301 from 
Table 1 Strains and plasmids used in this study

\begin{tabular}{|c|c|c|}
\hline $\begin{array}{l}\text { Strains or } \\
\text { plasmids }\end{array}$ & Description & Sources \\
\hline \multicolumn{3}{|l|}{ S. erythraea } \\
\hline A226 & $\begin{array}{l}\text { CGMCC } 8279 \text {, an erythromycin } \\
\text { low producer }\end{array}$ & $\begin{array}{l}\text { China Pharmaceutica } \\
\text { Culture Collection }\end{array}$ \\
\hline$\triangle S A C E \_7301$ & A226 with SACE_7301 deleted & This study \\
\hline $\begin{array}{l}\triangle S A C E_{-} 7301 / \\
\text { pZMW }\end{array}$ & $\triangle S A C E \_7301$ carrying pZMW & This study \\
\hline $\begin{array}{l}\triangle S A C E \text { 7301/ } \\
\text { pZMW7301 }\end{array}$ & $\begin{array}{l}\triangle S A C E \_7301 \text { carrying } \\
\text { pZMW7301 }\end{array}$ & This study \\
\hline A226/pZMW & A226 carrying pZMW & This study \\
\hline A226/pZMW7301 & A226 carrying pZMW7301 & This study \\
\hline A226/7301 & A226 carrying pSET152-7301 & This study \\
\hline$A 226 / 2 \times 7301$ & A226 carrying pSET152-2×7301 & This study \\
\hline A226/3×7301 & A226 carrying pSET152-3×7301 & This study \\
\hline WB & $\begin{array}{l}\text { CGMCC } 8280 \text {, an erythromycin } \\
\text { industrial overproducer }\end{array}$ & $\begin{array}{l}\text { Anhui Wanbei } \\
\text { Pharmaceutical Co., } \\
\text { Ltd. }\end{array}$ \\
\hline WB/7301 & WB carrying pSET152-7301 & This study \\
\hline $\mathrm{WB} / 2 \times 7301$ & WB carrying pSET152-2×7301 & This study \\
\hline $\mathrm{WB} / 3 \times 7301$ & WB carrying pSET152-3×7301 & This study \\
\hline \multicolumn{3}{|l|}{ E. coli } \\
\hline $\mathrm{DH} 5 \mathrm{a}$ & F recA lacZM15 & {$[40]$} \\
\hline BL21(DE3) & $\begin{array}{l}\text { F-ompT hsdSB (rB-mB-) gal dcm } \\
\text { (DE3) }\end{array}$ & Novagen \\
\hline \multicolumn{3}{|l|}{ Plasmids } \\
\hline pUCTSR & $\begin{array}{l}\text { pUC18 derivative containing a } \\
1.36-k b \text { fragment of a } \\
\text { thiostrepton resistance cassette } \\
\text { in the BamHI/Smal sites }\end{array}$ & {$[41]$} \\
\hline pUCTSR- $\Delta 7301$ & $\begin{array}{l}\text { pUCTSR carrying two 1.5-kb } \\
\text { fragments of the flanking } \\
\text { sequence of SACE_7301 gene }\end{array}$ & This study \\
\hline pZMW & $\begin{array}{l}\text { E. coli-S. erythraea integrative } \\
\text { shuttle vector carrying PermE* }\end{array}$ & {$[42]$} \\
\hline pZMW7301 & pZMW carrying SACE_7301 & This study \\
\hline $\begin{array}{l}\text { pBluescript II } \\
\text { SK (+) }\end{array}$ & bla, lacZ orif1 & Stratagene \\
\hline pSK-7301 & $\begin{array}{l}\text { pBluescript II SK (+) carrying } \\
\text { SACE_7301 under the control } \\
\text { of PermE* }\end{array}$ & This study \\
\hline pSET152 & $\begin{array}{l}\text { E. coli-S. erythraea integrative } \\
\text { shuttle vector }\end{array}$ & [43] \\
\hline pSET152-7301 & $\begin{array}{l}\text { PSET152 carrying SACE_7301 } \\
\text { under the control of PermE* }\end{array}$ & This study \\
\hline pSET152-2×7301 & $\begin{array}{l}\text { pSET152 carrying two extra } \\
\text { copies of SACE_7301 under the } \\
\text { control of PermE* }\end{array}$ & This study \\
\hline pSET152-3×7301 & $\begin{array}{l}\text { pSET152 carrying three extra } \\
\text { copies of SACE_7301 under the } \\
\text { control of PermE* }\end{array}$ & This study \\
\hline pET22b & T7 promoter, His-tag, kan & Novagen \\
\hline pET22b-7301 & pET22b carrying SACE_7301 & This study \\
\hline pET22b-bldD & pET22b carrying bldD & This study \\
\hline
\end{tabular}

the plasmid pZMW7301 was amplified using the primer pair ermE-F and 7301-R (Table 2). The amplified product was cloned into pBluescript II SK (+) via $\mathrm{XbaI}$ and SpeI sites (Figure 4A), and the correct sequence was confirmed by DNA sequencing. The fragment digested with $\mathrm{XbaI}$ and SpeI was repeatedly subcloned into $\mathrm{XbaI}$ site of pSET152 to generate pSET152-7301, pSET152-2×7301 and pSET152-3×7301, respectively (Figure $4 \mathrm{~A})$. Those plasmids were successively introduced into S. erythraea A226 or WB, and corresponding $S A C E_{-}$7301-overexpressed strains were obtained by apramycin screening and PCR confirmation.

\section{Fermentation and erythromycin assay}

For flask fermentation of A226 and its derivatives, spores from R3M plates cultured for 4 days were incubated in $250 \mathrm{ml}$ baffled flasks containing $30 \mathrm{ml}$ TSB medium for 2 days at $30^{\circ} \mathrm{C}$. Then, $3 \mathrm{ml}$ cultures were inoculated into $250 \mathrm{ml}$ baffled flasks containing $30 \mathrm{ml} \mathrm{R} 5$ liquid fermentation medium [38], and shaken for 6 days at $30^{\circ} \mathrm{C}(220 \mathrm{rpm})$. For flask fermentation of the erythromycin high-yielding strain WB and its derivatives, a liquid industrial medium was used as previously described [32]. After $24 \mathrm{~h}$ fermentation, $0.3 \mathrm{ml}$ of n-propanol was added into the cultures and further incubated for 5 days at $30^{\circ} \mathrm{C}$ (220 rpm).

For bioreactor cultures of $\mathrm{WB}$ and its $S A C E_{-} 7301$-overexpressed strain $\mathrm{WB} / 3 \times 7301$, the fermentation experiments were carried out in a $5 \mathrm{~L}$ fermentor (Baoxing, Shanghai, China) containing $3 \mathrm{~L}$ liquid industrial medium. Seed cultures for the cultivation in bioreactors were prepared as described above, using $450 \mathrm{ml}$ industrial seed medium in $2 \mathrm{~L}$ baffled flasks. Bioreactors were inoculated with 30 vol.\% seed culture. Dissolved oxygen concentration and $\mathrm{pH}$ were monitored using autoclavable electrodes (Mettler Toledo, Switzerland). Dissolved oxygen was maintained about $50 \%$ with increasing agitation and aeration rate during the bioprocess, and the $\mathrm{pH}$ was kept at 7.0 to 7.2. Foaming was controlled by automatic addition of antifoam. Samples $(50 \mathrm{ml})$ were taken every $24 \mathrm{~h}$ for Er-A yield analysis with HPLC as described previously [44]. HPLC analysis was performed on Agilent 1260 HPLC system equipped with an Agilent Extend-C18 column $(5 \mu \mathrm{m}, 250 \times 4.6 \mathrm{~mm})$, which was equilibrated with $40 \%$ solution A (acetonitrile, chromatographic grade) and $60 \%$ solution $B$ (potassium dihydrogen phosphate, $0.032 \mathrm{~mol} / \mathrm{L}, \mathrm{pH}$ 6.8). An isocratic program was carried out at a flow rate of $1.0 \mathrm{ml} / \mathrm{min}$ at $29^{\circ} \mathrm{C}$ using UV detector at $210 \mathrm{~nm}$.

For bioassay-based analysis, A226, $\triangle S A C E \_7301$ and $\triangle b l d D$ were grown in $30 \mathrm{ml} \mathrm{R} 5$ liquid medium in $250 \mathrm{ml}$ flasks for 6 days at $30^{\circ} \mathrm{C}$. $5 \mu$ fermentation 
supernatants from those liquid cultures were added to LB agar plates, which were sprayed with an overnight culture of $B$. subtilis PUB110. The plates were incubated at $37^{\circ} \mathrm{C}$ for $12 \mathrm{~h}$, and the erythromycin production was estimated by detecting the growth-inhibition zones as previously demonstrated [31]. Moreover, Er-A produced by $\mathrm{A} 226, \mathrm{WB}$ and their derivatives were quantitatively analyzed by HPLC.

\section{Transcriptional analysis by quantitative real-time PCR (qRT-PCR)}

The relative transcriptional levels of eryAI and ermE, from erythromycin biosynthetic gene cluster [7], were determined by qRT-PCR analysis. Specific primers were designed as listed in Table 2. According to the manufacturer's instructions of a RNA extraction/purification kit (SBS), total
RNA was extracted from S. erythraea A226 and its derivatives after 2 or 4 days of growth on R5 agar medium [38]. Isolated RNA was treated with DNase I (MBI Fermentas), and reverse transcription was achieved using a cDNA synthesis kit (MBI Fermentas). Quantitative real time PCR reactions were performed on the Applied Biosystems Step-One Plus system with Maxima ${ }^{\text {Tw }}$ SYBR Green/ROX qPCR Master Mix (MBI Fermentas). The $h r d B$ gene encoding the major sigma factor in $S$. erythraea was used as an internal control, and relative quantification was evaluated using a comparative cycle threshold $\left(C_{T}\right)$ method [45].

Overexpression and purification of SACE_7301 and BIdD As the original $S A C E$-7301 gene could not be expressed in E. coli BL21 (DE3) initially, we optimized the N-terminal

Table 2 Primers used in this study

\begin{tabular}{|c|c|c|}
\hline Name & Sequence $\left(5^{\prime}-3^{\prime}\right)$ (restriction site with italic formatting) & Use \\
\hline 7301-P1 & CGCGAATTCGCATCCTCG AGCACTTCACCG (ECORI) & \multirow[t]{4}{*}{ Inactivation of SACE_7301 } \\
\hline 7301-P2 & CCCGGTACCCGGCTCCGGATGGAACCG (Kpnl) & \\
\hline 7301-P3 & CTCTCTAGACACCACGGCGGCCTGCCG GC (Xbal) & \\
\hline 7301-P4 & CCAAAGCTTGGAGTCGGCGCATGCTGGTCTC (HindIII) & \\
\hline 7301-P5 & AGACATATGATGAAGGCCGACGTGGAGCAC (Ndel) & \multirow{4}{*}{$\begin{array}{l}\text { Complementation and overexpression } \\
\text { of SACE_7301 }\end{array}$} \\
\hline 7301-P6 & GCAGATATCTCACTCCGGTIT CCAGTCGCG (ECORV) & \\
\hline apr-F & GCTCATCGGTCAGCTTCTCA & \\
\hline apr-R & TCGCATTCTTCGCATCCC & \\
\hline 7301-P7 & GCTGGGTGTACTCGAAGAACGA & \multirow[t]{2}{*}{ qRT-PCR analysis of SACE_7301 } \\
\hline 7301-P8 & TGGGGTCGAAGGAGGAGC & \\
\hline 7301-P9 & $\begin{array}{l}\text { AGACATATGATGAAAGCCGATGTGGAGCATTCTGATCGTCC } \\
\text { GCGTCCGCGTACCAAGCGGCTGCCGCGC (Ndel) }\end{array}$ & \multirow[t]{2}{*}{ Expression of SACE_7301 In E. coli } \\
\hline 7301-P10 & CCCAAGCTTCTCCGGTTTCCAGTCGCGGCC (HindIII) & \\
\hline bldD-F & AAACATATGATGGGCGACTACGCCAAGGCGCTGGG (Ndel) & \multirow[t]{2}{*}{ Expression of BldD In E. coli } \\
\hline bldD-R & TGTAAGCTTCTCCTCCCGGGCCGGGCGC (HindIII) & \\
\hline ermE-F & TAATCTAGAGCGAGTGTCCGTTCGAGTGG (Xbal) & \multirow{2}{*}{$\begin{array}{l}\text { Cloning of combined DNA fragment containing } \\
\text { PermE* and SACE_7301 }^{*}\end{array}$} \\
\hline $7301-\mathrm{R}$ & GCAACTAGTTCACTCCGGTTTCCAGTCGCG (Spel) & \\
\hline eryAl-P1 & CCGCTGATGCCGAACGAC & \multirow[t]{2}{*}{ qRT-PCR analysis of eryAl } \\
\hline eryAl-P2 & CACCCTTCCCCGCACTCTG & \\
\hline eryAl-P3 & CGGAGCATTTGCTCGCTTTCCAGG & \multirow[t]{2}{*}{ EMSA of eryAl promoter } \\
\hline eryAl-P4 & GCGTCCCCCTACTCGACGACCAC & \\
\hline ermE-P1 & CCTCCAGGCACCAGTCCAC & \multirow[t]{2}{*}{ qRT-PCR analysis of ermE } \\
\hline ermE-P2 & AGTCGTTGCGGGAGAAGCT & \\
\hline ermE-P3 & GCGAGTGTCCGTTCGAGTGGCGG & \multirow[t]{2}{*}{ EMSA of ermE promoter } \\
\hline ermE-P4 & CGCTGGATCCTACCAACCGGCAC & \\
\hline hrdB-F & GGTCACGCCGTAGACCTGGC & \multirow[t]{2}{*}{ qRT-PCR analysis of $h r d B$ as internal reference } \\
\hline hrdB-R & CGGTGTCGTTCACGCTGCTG & \\
\hline Test-F & GCCAGTGCCAAGCTTGGGCTGCAGGTCGAC & \multirow{2}{*}{$\begin{array}{l}\text { PCR analysis of the cassette containing } 3 \text { copie } \\
\text { of PermE*-SACE_7301 }\end{array}$} \\
\hline Test-R & GAATTCGATATCGCGCGCGGCCGCGGATCC & \\
\hline
\end{tabular}


codons during the amplification of SACE 7301 using the primers 7301-P9 and 7301-P10 (Table 2). The PCR product was cloned into NdeI and HindIII restriction sites of pET22b to generate pET22b-7301. Then, pET22b-7301 was introduced into E. coli BL21 (DE3) for protein production. Gene expression was induced with $1 \mathrm{mM}$ isopropylthiogalactoside (IPTG) for $5 \mathrm{~h}$ at $30^{\circ} \mathrm{C}$. Cells were harvested and followed by sonication treatment. Purification of $\mathrm{His}_{6}$-tagged protein was carried out by use of Ni-NTA Sepharose chromatography [46]. The concentration of purified protein was quantified by BCA assays, and its purity was judged by sodium dodecyl sulfate polyacrylamide gel electrophoresis (SDS-PAGE) analysis. Similarly, bldD was amplified with the primers bldD-F and bldD-R (Table 2), and expressed in E. coli BL21 (DE3).

\section{Electrophoretic mobility shift assays (EMSAs)}

EMSAs were carried out in the light of previously described methods [47]. Using the genomic DNA from $S$. erythraea A226 as a template, the promoter regions of eryAI and ermE were successively amplified by PCR with their respective primers (Table 2) labeled at $5^{\prime}$ end by the 6-isomer of carboxyfluorescein (6-FAM). Those labeled DNA fragments were independently mixed with purified $\mathrm{His}_{6}$-tagged SACE 7301 or BldD proteins. The binding reaction system consisted of $10 \mathrm{mM}$ Tris (pH 7.5), $5 \mathrm{mM}$ $\mathrm{MgCl}_{2}, 50 \mathrm{mM}$ EDTA, $60 \mathrm{mM} \mathrm{KCl}, 10 \mathrm{mM}$ DTT, 10\% glycerol, $150 \mathrm{ng}$ labeled probes and 0.5-4 $\mu \mathrm{M}$ purified SACE_7301 or BldD. For competitive inhibition of the binding reaction, $7.5 \mu \mathrm{g}$ unlabeled above-mentioned DNA fragments or $7.5 \mu \mathrm{g}$ poly dIdC were added into that reaction system, respectively. After incubation on ice for $10 \mathrm{~min}$, the reactants were run on an $8 \%$ TBE polyacrylamide gel (Bio-Rad) with $0.5 \times \mathrm{TBE}$ as a running buffer at $30 \mathrm{~mA}$ for $1 \mathrm{~h}$.

\section{Additional file}

Additional file 1: Figure S1. Purification of His $s_{6}$-tagged SACE _ 7301 and $\mathrm{BldD}$. Left lane $116 \mathrm{KDa}$ protein ladder. Figure S2. Time courses of dry weight of mycelia (DWM) of low-yielding S. erythraea strain A226, industrial overproducer WB, and their derivatives. Mean values of at least three replicates were shown, with the standard deviation indicated by error bars. Figure S3. Growth of A226 and A226/3×7301 treated with erythromycin. The two strains were cultured in the R5 liquid medium for 2 days by addition of four concentrations of erythromycin $(40 \mu \mathrm{g} / \mathrm{ml}, 80 \mu \mathrm{g} / \mathrm{ml}, 160$ $\mu \mathrm{g} / \mathrm{ml}$ and $320 \mu \mathrm{g} / \mathrm{ml}$ ), respectively, and their dry weights of mycelia (DWM) were successively measured. Mean values of at least three replicates were shown, with the standard deviation indicated by error bars. Figure S4. PCR analysis of the cassette containing 3 copies of PermE*-SACE_7301 from A226/3 $\times 7301$ using the primers Test- $F$ and Test- $R$. The specific primers flanking above-mentioned cassette of the plasmid pSET152-3×7301 were designed as shown in Table 2. A226/3×7301 was cultured in the R5 liquid medium for 6 days without apramycin. Lanes: M: 5, 000-bp DNA ladder, +: positive control (pSET152-3×7301 as a template), -: negative control (Genomic DNA of A226 as a template), 1 to 6: Genomic DNA of A226/ $3 \times 7301$ cultured for 1 to 6 days, respectively, 7: Genomic DNA of the mixture of A226/7301, A226/2×7301 and A226/3×7301 with equal amount.
Figure S5. Phylogenetic tree of SACE_7301 homologs by the neighborjoining method. The number at each node indicates the percentage of 1000 bootstrap replications

\section{Abbreviations}

Er-A: Erythromycin A; ery: erythromycin biosynthetic gene; TFR: TetR family transcriptional regulator; PKS: Polyketide synthase; LB: Luria-Bertani medium: tsr: thiostrepton resistance gene; qRT-PCR: quantitative real-time PCR; IPTG: Isopropylthiogalactoside; SDS-PAGE: Sodium dodecyl sulfate polyacrylamide gel electrophoresis; EMSA: Electrophoretic mobility shift assay; 6-FAM: 6-isomer of carboxyfluorescein.

\section{Competing interests}

The authors declare that they have no competing interests.

\section{Authors' contributions}

HW, MC, YM and JL carried out the molecular genetics experiments, shaker-level fermentation and erythromycin assay. WL performed fermentor-level cultivation with sampling and determined the Er-A yield. BZ, DW and LZ conceived of the study, participated in its design, performed the data analysis and drafted the manuscript. XH, YZ and BY contributed to the coordination. All authors read and approved the final manuscript.

\section{Acknowledgments}

This work was supported by the National Program on Key Basic Research Project (973 program, 2013CB734000), Open Funding Project of the State Key Laboratory of Bioreactor Engineering (2013), The National Natural Science Foundation of China $(31300081,30870069)$, The Natural Science Foundation of Anhui Province (1208085MC46), and The Initial Foundation of Doctoral Scientific Research in Anhui University (01001904). LZ is an Awardee for National Distinguished Young Scholar Program in China.

\section{Author details}

'Institute of Health Sciences, School of Life Sciences, Anhui University, Hefei 230601, China. ${ }^{2}$ CAS Key Laboratory of Pathogenic Microbiology \& Immunology, Institute of Microbiology, Chinese Academy of Sciences, Beijing 100101, China. ${ }^{3}$ State Key Laboratory of Bioreactor Engineering, East China University of Science \& Technology, Shanghai 200237, China. ${ }^{4}$ Beijing Institute of Cell Biotechnology, Beijing 100043, China.

Received: 29 June 2014 Accepted: 23 October 2014

Published online: 13 November 2014

\section{References}

1. Butler MS: Natural products to drugs: natural product-derived compounds in clinical trials. Nat Prod Rep 2008, 25(3):475-516.

2. Reeves AR, Brikun IA, Cernota WH, Leach BI, Gonzalez MC, Weber JM: Engineering of the methylmalonyl-CoA metabolite node of Saccharopolyspora erythraea for increased erythromycin production. Metab Eng 2007, 9(3):293-303.

3. Wang Y, Wang Y, Chu J, Zhuang Y, Zhang L, Zhang S: Improved production of erythromycin A by expression of a heterologous gene encoding S-adenosylmethionine synthetase. Appl Microbiol Biotechnol 2007, 75(4):837-842

4. Chen Y, Deng W, Wu J, Qian J, Chu J, Zhuang Y, Zhang S, Liu W: Genetic modulation of the overexpression of tailoring genes ery $K$ and ery $G$ leading to the improvement of erythromycin A purity and production in Saccharopolyspora erythraea fermentation. Appl Environ Microbiol 2008, 74(6):1820-1828.

5. Cortes J, Haydock SF, Roberts GA, Bevitt DJ, Leadlay PF: An unusually large multifunctional polypeptide in the erythromycin-producing polyketide synthase of Saccharopolyspora erythraea. Nature 1990, 348(6297):176-178.

6. Donadio S, Staver MJ, McAlpine JB, Swanson SJ, Katz L: Modular organization of genes required for complex polyketide biosynthesis. Science 1991, 252(5006):675-679.

7. Weber J, Leung J, Maine G, Potenz R, Paulus T, DeWitt J: Organization of a cluster of erythromycin genes in Saccharopolyspora erythraea. J Bacteriol 1990, 172(5):2372-2383.

8. Cane DE: Programming of erythromycin biosynthesis by a modular polyketide synthase. J Biol Chem 2010, 285(36):27517-27523. 
9. Reeves AR, English RS, Lampel JS, Post DA, Vanden Boom TJ: Transcriptional organization of the erythromycin biosynthetic gene cluster of Saccharopolyspora erythraea. J Bacteriol 1999, 181(22):7098-7106.

10. Lum AM, Huang J, Hutchinson CR, Kao CM: Reverse engineering of industrial pharmaceutical-producing actinomycete strains using DNA microarrays. Metab Eng 2004, 6(3):186-196.

11. Elliot M, Damji F, Passantino R, Chater K, Leskiw B: The bldD gene of Streptomyces coelicolor $A 3(2)$ : a regulatory gene involved in morphogenesis and antibiotic production. J Bacterio/ 1998, 180(6):1549-1555.

12. Chng C, Lum AM, Vroom JA, Kao CM: A key developmental regulator controls the synthesis of the antibiotic erythromycin in Saccharopolyspora erythraea. Proc Natl Acad Sci U S A 2008, 105(32):11346-11351.

13. Kirm B, Magdevska V, Tome M, Horvat M, Karnicar K, Petek M, Vidmar R, Baebler S, Jamnik P, Fujs S, Horvat J, Fonovic M, Turk B, Gruden K, Petkovic H, Kosec G: SACE_5599, a putative regulatory protein, is involved in morphological differentiation and erythromycin production in Saccharopolyspora erythraea. Microb Cell Fact 2013, 12(1):126.

14. Ramos JL, Martinez-Bueno M, Molina-Henares AJ, Teran W, Watanabe K, Zhang X, Gallegos MT, Brennan R, Tobes R: The TetR family of transcriptional repressors. Microbiol Mol Biol Rev 2005, 69(2):326-356.

15. Hillerich $B$, Westpheling J: A new TetR family transcriptional regulator required for morphogenesis in Streptomyces coelicolor. J Bacteriol 2008, 190(1):61-67.

16. Hirano S, Tanaka K, Ohnishi Y, Horinouchi S: Conditionally positive effect of the TetR-family transcriptional regulator AtrA on streptomycin production by Streptomyces griseus. Microbiology 2008, 154(3):905-914.

17. Tahlan $K, Y u Z, X u Y$, Davidson AR, Nodwell JR: Ligand recognition by ActR, a TetR-like regulator of actinorhodin export. J Mol Biol 2008, 383(4):753-761.

18. Duong CT, Lee HN, Choi SS, Lee SY, Kim ES: Functional Expression of SAV3818, a Putative TetR-Family Transcriptional Regulatory Gene from Streptomyces avermitilis, Stimulates Antibiotic Production in Streptomyces Species. J Microbiol Biotechnol 2009, 19(2):136-139.

19. Ou X, Zhang B, Zhang $L$, Zhao G, Ding $X$ : Characterization of $\operatorname{rrdA}$, a TetR family protein gene involved in the regulation of secondary metabolism in Streptomyces coelicolor. Appl Environ Microbiol 2009, 75(7):2158-2165.

20. Lee HN, Huang J, Im JH, Kim SH, Noh JH, Cohen SN, Kim ES: Putative TetR family transcriptional regulator SCO1712 encodes an antibiotic downregulator in Streptomyces coelicolor. Appl Environ Microbiol 2010, 76(9):3039-3043.

21. Xu D, Seghezzi N, Esnault C, Virolle MJ: Repression of antibiotic production and sporulation in Streptomyces coelicolor by overexpression of a TetR family transcriptional regulator. Appl Environ Microbiol 2010, 76(23):7741-7753.

22. Chen Y, Zhu H, Zheng G, Jiang W, Lu Y: Functional analysis of TetR-family regulator AmtRsav in Streptomyces avermitilis. Microbiology 2013 159(Pt 12):2571-2583.

23. Guo J, Zhang X, Luo S, He F, Chen Z, Wen Y, Li J: A Novel TetR Family Transcriptional Regulator, SAV576, Negatively Controls Avermectin Biosynthesis in Streptomyces avermitilis. PLoS One 2013, 8(8):e71330.

24. Hayashi T, Tanaka Y, Sakai N, Okada U, Yao M, Watanabe N, Tamura T, Tanaka I: SCO4008, a putative TetR transcriptional repressor from Streptomyces coelicolor A3(2), regulates transcription of sco4007 by multidrug recognition. J Mol Biol 2013, 425(18):3289-3300.

25. Liu $Y$, Yan $T$, Jiang $L$, Wen $Y$, Song $Y$, Chen $Z$, Li J: Characterization of SAV7471, a TetR-family transcriptional regulator involved in the regulation of coenzyme A metabolism in Streptomyces avermitilis. J Bacteriol 2013, 195(19):4365-4372.

26. He F, Liu W, Sun D, Luo S, Chen Z, Wen Y, Li J: Engineering of the TetR family transcriptional regulator SAV151 and its target genes increases avermectin production in Streptomyces avermitilis. App/ Microbiol Biotechnol 2014, 98(1):399-409.

27. Wei J, Tian Y, Niu G, Tan H: GouR, a TetR family transcriptional regulator, coordinates the biosynthesis and export of gougerotin in Streptomyces graminearus. Appl Environ Microbiol 2014, 80(2):714-722.

28. Guo J, Zhang X, Chen Z, Wen Y, Li J: Two Adjacent and Similar TetR Family Transcriptional Regulator Genes, SAV577 and SAV576, Co-Regulate Avermectin Production in Streptomyces avermitilis. PLoS One 2014, 9(6):e99224

29. Oliynyk M, Samborskyy M, Lester JB, Mironenko T, Scott N, Dickens S, Haydock SF, Leadlay PF: Complete genome sequence of the erythromycin-producing bacterium Saccharopolyspora erythraea NRRL23338. Nat Biotechnol 2007, 25(4):447-453.
30. Han S, Song P, Ren T, Huang X, Cao C, Zhang B: Identification of SACE_7040, a member of TetR family related to the morphological differentiation of Saccharopolyspora erythraea. Curr Microbiol 2011 63(2):121-125

31. Yin X, Xu X, Wu H, Yuan L, Huang X, Zhang B: SACE_0012, a TetR-family transcriptional regulator, affects the morphogenesis of Saccharopolyspora erythraea. Curr Microbiol 2013, 67(6):647-651.

32. Wu P, Pan H, Zhang C, Wu H, Yuan L, Huang X, Zhou Y, Ye BC, Weaver DT, Zhang L, Zhang B: SACE_3986, a TetR family transcriptional regulator, negatively controls erythromycin biosynthesis in Saccharopolyspora erythraea. J Ind Microbiol Biotechnol 2014, 41(7):1159-1167.

33. Cuthbertson L, Nodwell JR: The TetR family of regulators. Microbio/ Mol Biol Rev 2013, 77(3):440-475.

34. Yu Z, Reichheld SE, Savchenko A, Parkinson J, Davidson AR: A comprehensive analysis of structural and sequence conservation in the TetR family transcriptional regulators. J Mol Biol 2010, 400(4):847-864.

35. He JJ, Huang XD, Song P, Guo JH, Chen HP, Cao C, Zhang BC: Effect of bldD gene overexpression on erythromycin output and spore formation of Saccharopolyspora erythraea. Bull Acad Mil Med Sci 2010, 34(3):251-254.

36. Corre $C$ : In search of the missing ligands for TetR family regulators. Chem Biol 2013, 20(2):140-142.

37. Zhu T, Cheng X, Liu Y, Deng Z, You D: Deciphering and engineering of the final step halogenase for improved chlortetracycline biosynthesis in industrial Streptomyces aureofaciens. Metab Eng 2013, 19:69-78.

38. Kieser T, Bibb MJ, Buttner MJ, Chater KF, Hopwood DA: Practical Streptomyces Genetics. Norwich: The John Innes Foundation; 2000.

39. Summers RG, Donadio S, Staver MJ, Wendt-Pienkowski E, Hutchinson CR, Katz $L$ : Sequencing and mutagenesis of genes from the erythromycin biosynthetic gene cluster of Saccharopolyspora erythraea that are involved in L-mycarose and D-desosamine production. Microbiology 1997, 143(Pt 10):3251-3262.

40. Sambrook J, Russell DW: Molecular Cloning: A Laboratory Manual. 3rd edition. New York: Cold Spring Harbor Laboratory; 2001.

41. Liu H, Huang XD, Liu DQ, Zhao W, Fan W, Han S, Zhang BC: Rapid chromosomic gene inactivating technology of Saccharopolyspora erythraea. Bull Acad Mil Med Sci 2009, 33(4):365-369.

42. Zhang BC, Li LL, Yu XQ, Liu CX, Wang YG, He BQ, Ma QJ: Construction of Saccharopolyspora erythraea expression vector pZMW. Bull Acad Mil Med Sci 2003, 27(3):176-179.

43. Bierman M, Logan R, O'Brien K, Seno ET, Rao RN, Schoner BE: Plasmid cloning vectors for the conjugal transfer of DNA from Escherichia coli to Streptomyces spp. Gene 1992, 116(1):43-49.

44. Tsuji K, Goetz JF: HPLC as a rapid means of monitoring erythromycin and tetracycline fermentation processes. J Antibiot (Tokyo) 1978, 31(4):302-308.

45. Livak K, Schmittgenb T: Analysis of relative gene expression data using real-time quantitative PCR and the $2{ }^{\Delta \Delta C T}$. Methods 2001, 25(4):402-408,

46. Porath J, Carlsson J, Olsson I, Belfrage G: Metal chelate affinity chromatography, a new approach to protein fractionation. Nature 1975, 258(5536):598-599.

47. Hellman LM, Fried MG: Electrophoretic mobility shift assay (EMSA) for detecting protein-nucleic acid interactions. Nat Protoc 2007, 2(8):1849-1861.

\section{doi:10.1186/s12934-014-0158-4}

Cite this article as: Wu et al.: Dissecting and engineering of the TetR family regulator SACE 7301 for enhanced erythromycin production in Saccharopolyspora erythraea. Microbial Cell Factories 2014 13:158. 